\title{
Strong Resonance Investigation and Suppression in PMSG integrated Power Systems
}

\author{
Jianqiang LUO, Siqi BU*, Jiaxin WEN, Qian HU, Yong HU, and Qi WANG \\ Department of Electrical Engineering, The Hong Kong Polytechnic University, Hung Hom, Hong Kong SAR, China
}

\begin{abstract}
Permanent magnet synchronous generators (PMSGs) with full converters have been widely used in wind power generation due to its superior flexibility and controllability. However, under some circumstance, the oscillation modes of PMSG (POMs) may excite strong resonance with the electromechanical oscillation modes (EOMs) of the power system that degrades the power system small signal stability. In this paper, A two-open-loop subsystem model is firstly derived to analyze the oscillation modes. Then the POMs are investigated with modal analysis, the relationship between POMs and related controllers are clarified. On this basis, the strong resonance between PMSG and the external power system is revealed and identified. Furthermore, a five-step parameter tuning method is proposed to relocate the position of POM as well as suppress the strong resonance. Both modal analysis and time-domain simulations validate the effectiveness of the proposed method.
\end{abstract}

\section{Introduction}

In the last several decades, wind power generation grows very fast over the world due to the requirements of carbon reduction. To achieve better controllability, various control schemes have been proposed in the wind power generation to improve dynamic performance [1-5]. Full converter-based wind power generation (FCWG) $[1$, $6,7]$, which uses full scale converters to connect the variable speed wind generators (VSWGs) with the power grid, becomes very popular these years due to the wide application and cost reduction of power electronics. Among FCWG, the direct-driven permanent magnet synchronous generator (PMSG) is a very promising wind generation technology, which may dominate the wind power market in the next decade.

However, unlike the conventional synchronous generators (CSGs), the integration of wind power generators does bring problems, such as inertia reduction, dynamic interaction complexity, protection inaccuracy $[6$, 8-10]. The unexpected concern on power system stability also draws great attention in this field $[6,11,12]$. Since FCWG has completely different physical structure from CSGs, its impact mechanism on power system small signal stability needs to be further clarified. Early works in this field have made some valuable progress. A model reduction strategy proposed in [13] can reduce the model complexity and improve the computational efficiency of a large-scale power system with increasing penetration of wind power generation. [14] presents a stationary $\alpha \beta$ frame impedance model to predict the stability impact of PLL and its coupling effect. The mechanism of the system instability conducted in [15] uses "positive feedback effect" between the electrical subsystem and the control subsystem to explain the dynamic process of instability brought by power converters.

As for other published works, the impact of the integration of FCWG on power system small signal stability with can be assessed from two aspects, i.e., the power flow changes and the dynamic interaction. [6] performed modal analysis to evaluate the overall impact, whilst [11] and[16] investigate the impact of VSWGs on small signal stability by using damping torque analysis, which can be a considerable improvement in understanding the overall impact.

In this paper, the strong modal resonance that threats the power system small signal stability is studied. A fivestep parameter optimization method is proposed to tune the open-loop oscillation mode of PMSG. Since the POM tuning objective function is quite complex, which cannot be obtained analytically, the particle swarm optimization (PSO) algorithm is applied to optimize controller parameters of PMSG. PSO algorithm can evaluate the quality of the solution through fitness and has demonstrated its advantages in solving practical problems such as easy implementation, high precision and fast convergence [17].

The rest of this paper is organized as follows. Section 2 introduces the modeling and linearization of PMSG and the external power system. Section 3 presents the strong modal resonance between PMSG and the external power system. A POM tuning method is also proposed to optimize the controller parameters to suppress the strong modal resonance. Section 4 gives a case study to demonstrate the proposed optimization method, while Section 5 concludes this paper.

Corresponding author: siqi.bu@ polyu.edu.hk 


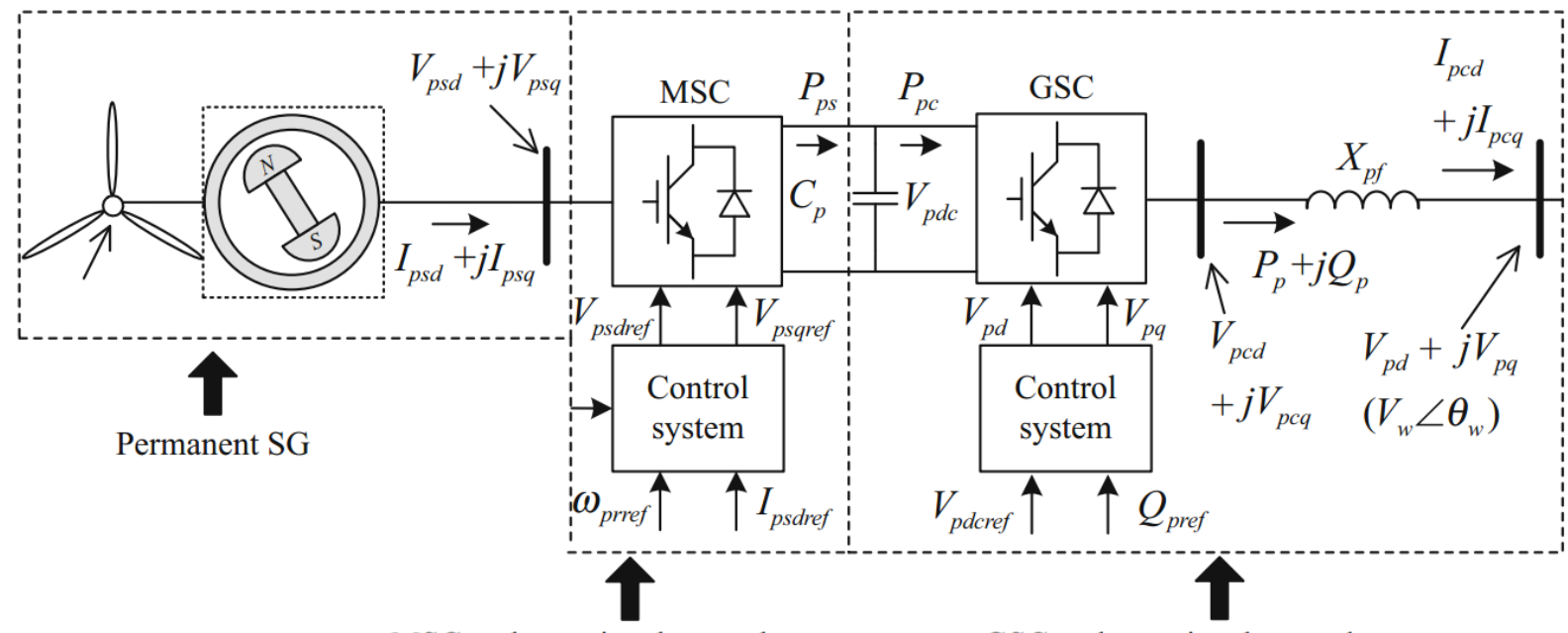

MSC and associated control system

GSC and associated control system

Fig. 1. Configuration of a PMSG connected the power system

\section{Modeling of PMSG integrated Power System}

\subsection{Linearization of PMSG}

A typical PMSG with full converters is shown in Fig. 1. There are four main parts: 1) The permanent magnet synchronous generator; 2) The machine side controller (MSC) and the associated control system; 3) The DClink, the grid side converter (GSC) and the associated control system; 4) The synchronous reference frame phase-locked loop (SRF-PLL) which keeps the synchronization with the power system.

The linearization equations for PMSG under $\mathrm{x}-\mathrm{y}$ coordinates system can be expressed as below

$$
\left\{\begin{array}{l}
\frac{d}{d t} \Delta \mathbf{X}_{\mathrm{pp}}=\mathbf{A}_{\mathrm{gp}} \Delta \mathbf{X}_{\mathrm{pp}}+\mathbf{B}_{\mathrm{gp}} \Delta \mathbf{V} \\
\Delta \mathbf{I}=\mathbf{C}_{\mathrm{gp}} \Delta \mathbf{X}_{\mathrm{pp}}
\end{array}\right.
$$

where $\quad \Delta \mathbf{V}=\left[\begin{array}{ll}\Delta V_{x} & \Delta V_{y}\end{array}\right]^{T}, \quad \Delta \mathbf{I}=\left[\begin{array}{ll}\Delta I_{x} & \Delta I_{y}\end{array}\right]^{T}$,

$$
\mathbf{A}_{\mathrm{gp}}=\mathbf{A}_{\mathbf{p p}}+\mathbf{B}_{\mathbf{p p}} R_{v g}, \mathbf{B}_{\mathrm{gp}}=B_{p p} T_{g 0},
$$

$\mathbf{C}_{\mathrm{gp}}=T_{g 0}^{-1}\left(C_{p p}-R_{I g}\right)=T_{g 0}^{-1}\left[\begin{array}{lllllllllllllll}0 & 0 & 0 & 0 & 0 & 0 & 1 & 0 & 0 & 0 & 0 & 0 & 0 & 0 & -I_{p c q 0} \\ 0 & 0 & 0 & 0 & 0 & 0 & 0 & 1 & 0 & 0 & 0 & 0 & 0 & 0 & I_{p c d 0}\end{array}\right]$

Hence, the transfer function of PMSG can be obtained,

$$
\Delta \mathbf{I}=\mathbf{C}_{\mathbf{g p}}\left(s I-\mathbf{A}_{\mathbf{g p}}\right)^{-1} \mathbf{B}_{\mathbf{g p}} \Delta \mathbf{V}=H(s) \Delta \mathbf{V}
$$

$$
\text { where } H(s)=\mathbf{C}_{\mathbf{g p}}\left(s I-\mathbf{A}_{\mathbf{g p}}\right)^{-1} \mathbf{B}_{\mathbf{g p}}=\left[\begin{array}{ll}
h_{11}(s) & h_{12}(s) \\
h_{21}(s) & h_{22}(s)
\end{array}\right] \text {. }
$$

\subsection{Linearization of External Power System}

The state space model for $\mathrm{N}$ generators in the rest of the power system can be written as

$$
\left\{\begin{array}{l}
\frac{d}{d t} \Delta X_{g}=A_{g} \Delta X_{g}+B_{g} \Delta V_{g} \\
\Delta I_{g}=C_{g} \Delta X_{g}+D_{g} \Delta V_{g}
\end{array}\right.
$$

The network equation is

$$
\left[\begin{array}{l}
I_{g} \\
I \\
I_{N}
\end{array}\right]=\left[\begin{array}{lll}
Y_{g g} & Y_{g w} & Y_{g n} \\
Y_{w g} & Y_{w w} & Y_{w n} \\
Y_{n g} & Y_{n w} & Y_{n n}
\end{array}\right]\left[\begin{array}{l}
V_{g} \\
V \\
V_{N}
\end{array}\right]=Y\left[\begin{array}{l}
V_{g} \\
V \\
V_{N}
\end{array}\right]
$$

where $I_{g}, V_{g}$ is the SG terminal current injection and bus voltage at the connecting point; $I, V$ is the output current and voltage at the PCC for PMSG, which is modeled as a current source; $I_{N}, V_{N}$ is the current injection and voltage at other buses in the network; $Y$ is the admittance matrix of the multi-machine power system.

Linearize $\mathrm{Eq}(4)$, it can have

$$
\left[\begin{array}{c}
\Delta I_{g} \\
\Delta I \\
0
\end{array}\right]=Y\left[\begin{array}{l}
\Delta V_{g} \\
\Delta V \\
\Delta V_{N}
\end{array}\right]=\left[\begin{array}{lll}
Y_{g g} & Y_{g w} & Y_{g n} \\
Y_{w g} & Y_{w w} & Y_{w n} \\
Y_{n g} & Y_{n w} & Y_{n n}
\end{array}\right]\left[\begin{array}{l}
\Delta V_{g} \\
\Delta V \\
\Delta V_{N}
\end{array}\right]
$$

Hence, the network equation can be rewritten as

$$
\left[\begin{array}{c}
\Delta I_{g} \\
\Delta I
\end{array}\right]=\left[\begin{array}{ll}
Y_{g g N} & Y_{g w N} \\
Y_{w g N} & Y_{w w N}
\end{array}\right]\left[\begin{array}{c}
\Delta V_{g} \\
\Delta V
\end{array}\right]
$$

where $Y_{g g N}=\left(Y_{g g}-Y_{g n} Y_{n n}^{-1} Y_{n g}\right), Y_{g w N}=Y_{g w}-Y_{g n} Y_{n n}^{-1} Y_{n w}$, $Y_{w g N}=\left(Y_{w g}-Y_{w n} Y_{n n}^{-1} Y_{n g}\right), Y_{w w N}=\left(Y_{w w}-Y_{w n} Y_{n n}^{-1} Y_{n w}\right)$.

Hence, the linearization equations of the external power system can be expressed as

$$
\left\{\begin{array}{l}
\frac{d}{d t} \Delta \mathbf{X}_{\mathrm{g}}=\mathbf{A}_{\mathrm{gT}} \Delta \mathbf{X}_{\mathrm{g}}+\mathbf{B}_{\mathrm{gT}} \Delta I \\
\Delta V=\mathbf{C}_{\mathrm{gT}} \Delta \mathbf{X}_{\mathrm{g}}+d_{I} \Delta I
\end{array}\right.
$$

where $\mathbf{A}_{\mathrm{gT}}=A_{g}+B_{g}\left(Y_{g g N}-Y_{g w N} Y_{w w N}^{-1} Y_{w g N}-D_{g}\right)^{-1} C_{g}$,

$\mathbf{B}_{\mathrm{gT}}=-B_{g}\left(Y_{g g N}-Y_{g w N} Y_{w w N}^{-1} Y_{w g N}-D_{g}\right)^{-1} Y_{g w N} Y_{w w N}^{-1}$,

$\mathbf{C}_{\mathrm{gT}}=-Y_{w w N}^{-1} Y_{w g N}\left(Y_{g g N}-Y_{g w N} Y_{w w N}^{-1} Y_{w g N}-D_{g}\right)^{-1} C_{g}$,

$d_{I}=Y_{w w N}^{-1}+Y_{w w N}^{-1} Y_{w g N}\left(Y_{g g N}-Y_{g w N} Y_{w w N}^{-1} Y_{w g N}-D_{g}\right)^{-1} Y_{g w N} Y_{w w N}^{-1}$. 


\subsection{Closed-loop Model of Entire Power System}

Combine $\mathrm{Eq}(1)$ and $\mathrm{Eq}(7)$, it can have

$$
\left\{\begin{array}{l}
\frac{d}{d t} \Delta \mathbf{X}_{\mathrm{g}}=\mathbf{A}_{\mathrm{gT}} \Delta \mathbf{X}_{\mathrm{g}}+\mathbf{B}_{\mathrm{gT}} C_{g p} \Delta X_{p p} \\
\frac{d}{d t} \Delta X_{p p}=B_{g p} \mathbf{C}_{\mathrm{gT}} \Delta \mathbf{X}_{\mathrm{g}}+\left(A_{g p}+B_{g p} d_{I} C_{g p}\right) \Delta X_{p p}
\end{array}\right.
$$

Hence, the closed-loop interconnected model of the entire power system is obtained as

$$
\frac{d}{d t}\left[\begin{array}{c}
\Delta \mathbf{X}_{\mathrm{g}} \\
\Delta \mathbf{X}_{\mathbf{p p}}
\end{array}\right]=\left[\begin{array}{cc}
\mathbf{A}_{\mathrm{gT}} & \mathbf{B}_{\mathrm{gT}} C_{g p} \\
B_{g p} \mathbf{C}_{\mathrm{gT}} & A_{g p}+B_{g p} d_{I} C_{g p}
\end{array}\right]\left[\begin{array}{c}
\Delta \mathbf{X}_{\mathrm{g}} \\
\Delta \mathbf{X}_{\mathrm{pp}}
\end{array}\right]
$$

\section{Resonance Identification and Suppression}

\subsection{Strong Modal Resonance}

Based on the modeling in Section 2, the total system can be divided into two subsystems. The bus voltage variation at PCC can be regarded as the input of PMSG, and the output is current injection variation that interacts with the external power system.

Denote $\lambda_{\mathrm{g}}$ as the critical electromechanical oscillation mode (EOM) of the open-loop power system. Denote $\lambda_{\mathrm{h}}$ as the open loop-mode of PMSG (POM) which has the largest impact on the critical EOM of the power system. The distance between $\lambda_{\mathrm{g}}$ and $\lambda_{\mathrm{h}}$ is $\Delta d=\lambda_{\mathrm{h}}-\lambda_{\mathrm{g}}$.

Based on $\operatorname{Eq}(1)$ and $\operatorname{Eq}(7)$, the transfer function of PMSG and the power system can be expressed as

$$
\begin{aligned}
& G(s)=\mathbf{C}_{\mathrm{gT}}\left(s I-\mathbf{A}_{\mathrm{gT}}\right)^{-1} \mathbf{B}_{\mathrm{gT}}+d_{I}=\frac{1}{\left(s-\lambda_{g}\right)}\left[\begin{array}{ll}
g_{11}(s) & g_{12}(s) \\
g_{21}(s) & g_{22}(s)
\end{array}\right] \\
& H(s)=\mathbf{C}_{\mathrm{gp}}\left(s I-\mathbf{A}_{\mathrm{gp}}\right)^{-1} \mathbf{B}_{\mathrm{gp}}+\mathbf{D}_{\mathrm{gp}}=\frac{1}{\left(s-\lambda_{h}\right)}\left[\begin{array}{ll}
h_{11}(s) & h_{12}(s) \\
h_{21}(s) & h_{22}(s)
\end{array}\right]
\end{aligned}
$$

The characteristic equation of the closed-loop interconnected power system is

$$
|G(s) H(s)-I|=0
$$

Denote $\hat{\lambda}_{\mathrm{g}}$ and $\hat{\lambda}_{\mathrm{h}}$ as the solutions of $\operatorname{Eq}(9)$ corresponding to $\lambda_{\mathrm{g}}$ and $\lambda_{\mathrm{h}}$. According to open-loop modal resonance theory proposed in [18], when $\Delta \mathrm{d} \approx 0$, i.e. $\lambda_{\mathrm{h}} \approx \lambda_{\mathrm{g}}, \hat{\lambda}_{\mathrm{g}}$ and $\hat{\lambda}_{\mathrm{h}}$ tend to move the right side and the left side of $\lambda_{\mathrm{g}}$ and $\lambda_{\mathrm{h}}$. The direction of movement of $\hat{\lambda}_{\mathrm{g}}$ and $\hat{\lambda}_{\mathrm{h}}$ depends on the relative location of $\lambda_{\mathrm{g}}$ and $\lambda_{\mathrm{h}}$.
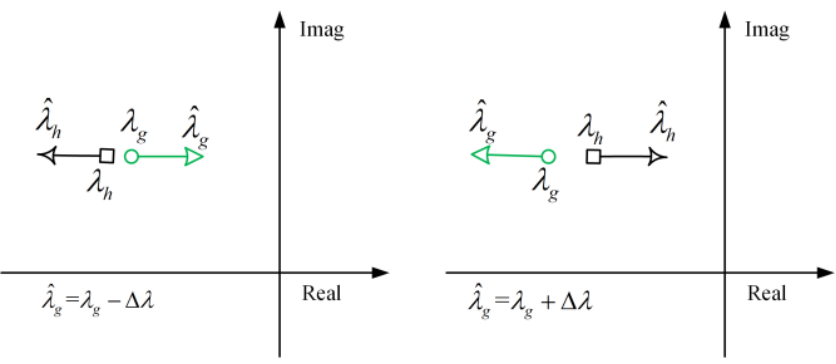

Fig. 2. The possible movements of modal resonance
For example, if $\lambda_{\mathrm{h}}$ is at the right of $\lambda_{\mathrm{g}}$, the system electromechanical mode may move to the right side, the PMSG mode may move to the left side, and vice versa, as shown in Fig. 2.

From the discussion above, it can be concluded that the relative position of POM and EOM may lead to strong modal resonance, and one of the closed-loop modes may deteriorate.

\subsection{Resonance Suppression with POM Tuning}

To reduce the negative effect on system small signal stability, the POM tuning method is proposed to move the POM away from EOM. There are five major steps in POM tuning:

(1) Identify the EOM;

(2) Identify the POMs and their related state variables;

(3) Compare the distance between POM and EOM, decide which of the POMs need to be tuned;

(4) Based on Step 2\&3, tune the parameters of controllers according to the related state variables and associated POM;

(5) Use time domain simulation to verify the effectiveness of POM tuning.

To obtain the feasible parameters of POM, the question can be redefined as

$$
\begin{aligned}
& \text { minimize } f\left(x_{i}\right)=\sum_{t_{0}}^{t_{1}} \Delta P_{e}\left(x_{i}\right) \\
& \text { subject to } x_{l} \leq x_{i} \leq x_{h} \quad l, i, h=1, \ldots, m
\end{aligned}
$$

where $\Delta P_{e}$ is the active power variation on critical power tie line, $x_{i}$ represent the parameters of PMSG controllers to be tuned, $x_{l}$ and $x_{h}$ are the low boundary and high boundary of $x_{i}, f\left(x_{i}\right)$ is the closed-loop system dynamic response error function.

The objective function $f\left(x_{i}\right)$ is proposed to minimize the critical electromechanical power oscillation in the external power system, which can be measured at critical bus. Once the minimal $f\left(x_{i}\right)$ is achieved, the power oscillation is suppressed at the optimal level. Since the objective function is not explicit, the traditional PSO algorithm in [17]. is implemented to solve this constrained optimization problem.

\section{Case Study}

An example system is built as shown in Fig. 3. A PMSG is connected to the single machine infinite bus (SMIB) system.

The five-step tuning is demonstrated as follows.

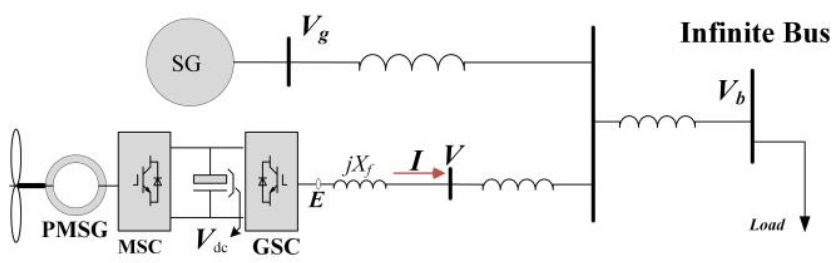

Fig. 3. PMSG-SMIB system 


\section{Step 1: Identify EOM}

Consider the SMIB system, the open loop EOM is identified to be $\lambda_{g}=-0.3148+4.5190 \mathrm{i}$ through modal analysis.

\section{Step 2: Identify POMs}

Modal analysis is carried out on the open loop PMSG model with power flow profile at PCC, and thus the typical modes can be identified by employing participation factors with associated variables, as shown in Table 1.

Table 1. The open-loop modes of PMSG

\begin{tabular}{|c|c|c|c|c|}
\hline Mode & Eigenvalue & $\begin{array}{c}\text { Frequen } \\
\mathbf{c y}(\mathbf{H z})\end{array}$ & $\begin{array}{c}\text { Damping } \\
\text { ratio }\end{array}$ & $\begin{array}{c}\text { Associated } \\
\text { variables }\end{array}$ \\
\hline POM1 & $\begin{array}{c}-2.5000 \pm \\
22.2205 \mathrm{i}\end{array}$ & 3.5365 & 0.1118 & $\Delta \psi_{p s d} x_{p 3}$ \\
\hline POM2 & $\begin{array}{c}-2.1846 \pm \\
22.2055 \mathrm{i}\end{array}$ & 3.5341 & 0.0979 & $\Delta \psi_{p s q} \Delta x_{p 2}$ \\
\hline POM3 & $\begin{array}{c}-0.3154 \pm \\
1.5528 \mathrm{i}\end{array}$ & 0.2471 & 0.1990 & $\Delta \omega_{p r} \Delta x_{p 1}$ \\
\hline POM4 & $\begin{array}{c}-12.4730 \pm \\
69.6261 \mathrm{i}\end{array}$ & 11.0813 & 0.1763 & $\Delta I_{p c d} \Delta x_{p 5}$ \\
\hline POM5 & $\begin{array}{c}-22.4212 \pm \\
97.3898 \mathrm{i}\end{array}$ & 15.5001 & 0.2244 & $\Delta I_{p c q} \Delta x_{p 7}$ \\
\hline POM6 & $\begin{array}{c}-0.0371 \pm \\
0.8123 \mathrm{i}\end{array}$ & 0.1293 & 0.0456 & $\Delta V_{p d c} \Delta x_{p 4}$ \\
\hline POM7 & -4.9684 & 0.0000 & 1.0000 & $\Delta x_{p 6}$ \\
\hline POM8 & $\begin{array}{c}-0.3145 \pm \\
4.5138 \mathrm{i}\end{array}$ & 0.7184 & 0.0695 & $\Delta x_{p l l} \Delta \theta_{p l l}$ \\
\hline
\end{tabular}

\section{Step 3: Resonance Mode Identification}

From Table 1, it is easy to find that the EOM is very close to the one of the POMs, i.e. POM8 (the PLL mode). Then POM tuning is to optimize POM8.

\section{Step 4: Parameter optimization}

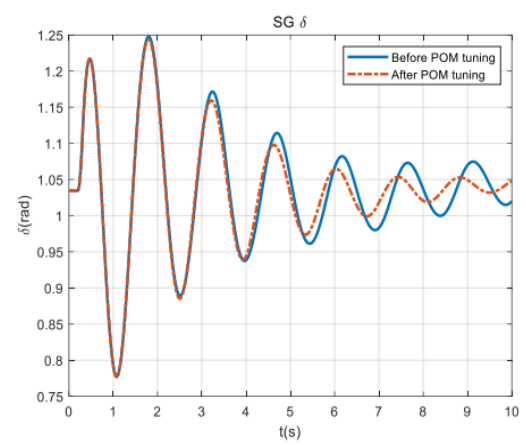

(a)

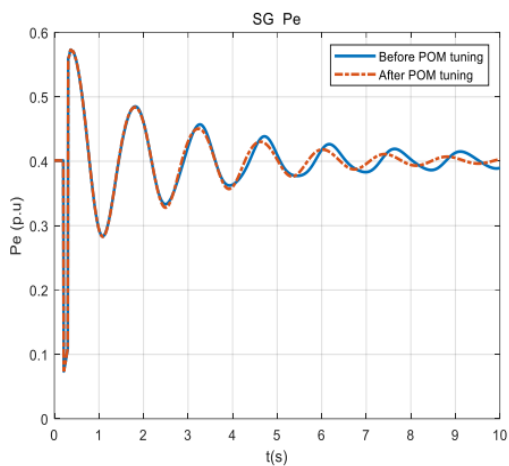

(d)

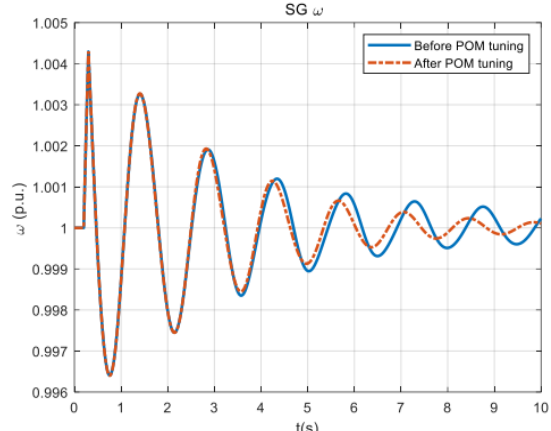

(b)

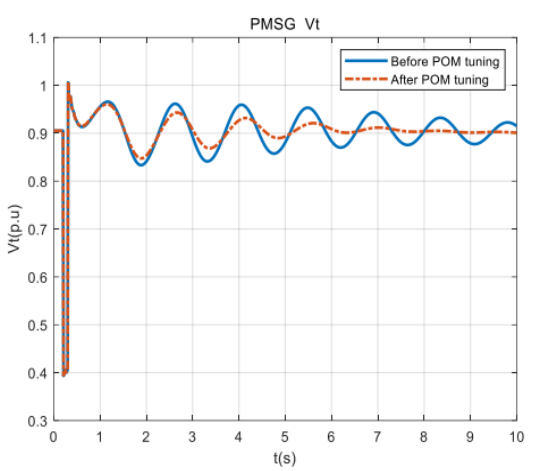

(e)

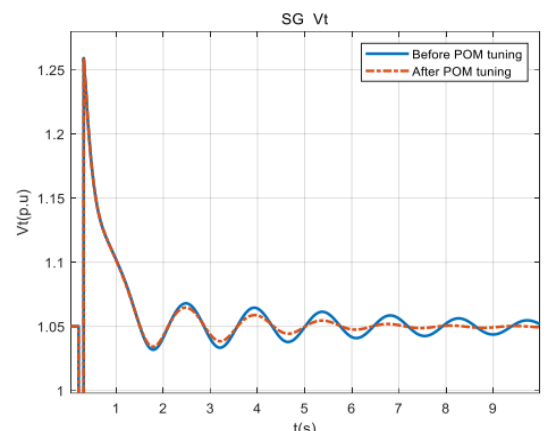

(c)

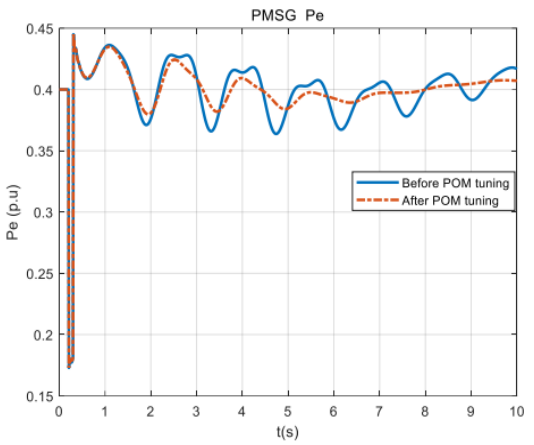

(f)

Fig. 4. Simulation comparison verification on POM tuning 


\section{Conclusion}

In this paper, the strong modal resonance between PMSG and the external power system is analyzed. A POM tuning based on parameter optimization is proposed to suppress the negative impact of modal resonance on power system small signal stability. Once the objective POM is identified, the constrained optimization problem can be determined to tune the related controller parameters. The PSO algorithm is employed to optimize the dynamic performance error function and achieve a larger damping ratio of the closed-loop power system. The five-step case study has demonstrated the effectiveness of the proposed method. Therefore, better damping characteristics of EOM can be achieved with the proposed POM tuning method without auxiliary damping equipment installed, which is more economical for system operators.

\section{Acknowledgment}

The authors would like to acknowledge the support from Department of Electrical Engineering, The Hong Kong Polytechnic University for the Start-up Fund Research Project (1-ZE68), Hong Kong Research Grant Council for the Research Projects (25203917), (15200418) and (15219619), and National Natural Science Foundation of China for the Research Project (51807171).

\section{References}

1 O. Carranza, E. Figueres, G. Garcerá, and R. Gonzalez-Medina, "Analysis of the control structure of wind energy generation systems based on a permanent magnet synchronous generator," Applied Energy, vol. 103, pp. 522-538, (2013)

2 D. Gautam, L. Goel, R. Ayyanar, V. Vittal, and T. Harbour, "Control Strategy to Mitigate the Impact of Reduced Inertia Due to Doubly Fed Induction Generators on Large Power Systems," IEEE Transactions on Power Systems, vol. 26, no. 1, pp. 214-224, (2011)

3 Y. Wang, J. Meng, X. Zhang, and L. Xu, "Control of PMSG-Based Wind Turbines for System Inertial Response and Power Oscillation Damping," IEEE Transactions on Sustainable Energy, vol. 6, no. 2, pp. 565-574, (2015)

4 Y. Li, Z. Xu, and K. P. Wong, "Advanced Control Strategies of PMSG-Based Wind Turbines for System Inertia Support," IEEE Transactions on Power Systems, vol. 32, no. 4, pp. 3027-3037, (2017)

5 J. Fang, P. Lin, H. Li, Y. Yang, and Y. Tang, "An Improved Virtual Inertia Control for Three-Phase Voltage Source Converters Connected to a Weak Grid," IEEE Transactions on Power Electronics, pp. 1-1, (2018)

6 J. Quintero, V. Vittal, G. T. Heydt, and H. Zhang, "The Impact of Increased Penetration of Converter Control-Based Generators on Power System Modes of Oscillation," IEEE Transactions on Power Systems, vol. 29, no. 5, pp. 2248-2256, (2014)
7 J. He, L. Huang, C. Zhu, and H. Xin, "Frequency Support from PMSG-Based Wind Turbines with Reduced DC-Link Voltage Fluctuations," China Electrotechnical Society Transactions on Electrical Machines and Systems, vol. 2, no. 3, pp. 296-302, (2018)

8 Q. Hu, L. Fu, F. Ma, and F. Ji, "Large Signal Synchronizing Instability of PLL-Based VSC Connected to Weak AC Grid," IEEE Transactions on Power Systems, pp. 1-1, (2019)

9 L. P. Kunjumuhammed, B. C. Pal, R. Gupta, and K. J. Dyke, "Stability Analysis of a PMSG-Based Large Offshore Wind Farm Connected to a VSC-HVDC," IEEE Transactions on Energy Conversion, vol. 32, no. 3, pp. 1166-1176, (2017)

10 J. Ekanayake and N. Jenkins, "Comparison of the Response of Doubly Fed and Fixed-Speed Induction Generator Wind Turbines to Changes in Network Frequency," IEEE Transactions on Energy Conversion, vol. 19, no. 4, pp. 800-802, (2004)

11 W. Du, J. Bi, J. Cao, and H. F. Wang, "A Method to Examine the Impact of Grid Connection of the DFIGs on Power System Electromechanical Oscillation Modes," IEEE Transactions on Power Systems, vol. 31, no. 5, pp. 3775-3784, (2016)

12 F. Wu, X. P. Zhang, and P. Ju, "Small signal stability analysis and control of the wind turbine with the direct-drive permanent magnet generator integrated to the grid," Electric Power Systems Research, vol. 79, no. 12, pp. 1661-1667, (2009)

13 S. W. Xia, S. Q. Bu, X. Zhang, Y. Xu, B. Zhou, and J. B. Zhu, "Model reduction strategy of doubly-fed induction generator-based wind farms for power system small-signal rotor angle stability analysis," Applied Energy, vol. 222, pp. 608-620, (2018)

14 X. Wang, L. Harnefors, and F. Blaabjerg, "Unified Impedance Model of Grid-Connected VoltageSource Converters," IEEE Transactions on Power Electronics, vol. 33, no. 2, pp. 1775-1787, (2018)

15 S. Zhao and B. Shao, "An analytical method suitable for revealing the instability mechanism of power electronics dominated power systems," International Journal of Electrical Power \& Energy Systems, vol. 109, pp. 269-282, (2019)

16 S. Q. Bu, X. Zhang, J. B. Zhu, and X. Liu, "Comparison analysis on damping mechanisms of power systems with induction generator based wind power generation," International Journal of Electrical Power \& Energy Systems, vol. 97, pp. 250-261, (2018)

17 D. Cristian, C. Barbulescu, S. Kilyeni, and V. Popescu, "Particle swarm optimization techniques. Power systems applications," in 6th International Conference on Human System Interactions (HSI), (2013)

18 W. Du, J. Bi, and H. Wang, "Damping Degradation of Power System Low-Frequency Electromechanical Oscillations Caused by OpenLoop Modal Resonance," IEEE Transactions on Power Systems, vol. 33, no. 5, pp. 5072-5081, (2018) 